\title{
Modeling of a Modern Aircraft Through Calibration Techniques
}

\author{
Robert Thacker* \\ NASA Glenn Research Center, Cleveland, OH, 44135, USA \\ Nathaniel J. Blaesser ${ }^{\dagger}$ \\ NASA Langley Research Center, Hampton, VA, 23681, USA
}

\begin{abstract}
NASA is seeking a new baseline aircraft model to assess the state-of-the-art technology for aircraft noise, emissions, and fuel/energy consumption as an update to a 2005 baseline. The process of modeling engine and airframe models as a system has historically required many iterations at NASA between the airframe and engine models. A new internal process presented in this paper contains a method that simultaneously calibrates an airframe and engine model to known data to create an aircraft system model. The work presented in this paper proposes a new framework in creating new aircraft models for future NASA research. This approach is presented as a general outline applicable to any chosen commercial aircraft. As an applied example, the B737 MAX 8 aircraft is chosen as the integrated engine and airframe model subjected to calibration. Initial results show a close match to available data but further refinement in the process is necessary for this ongoing work.
\end{abstract}

\section{Nomenclature}

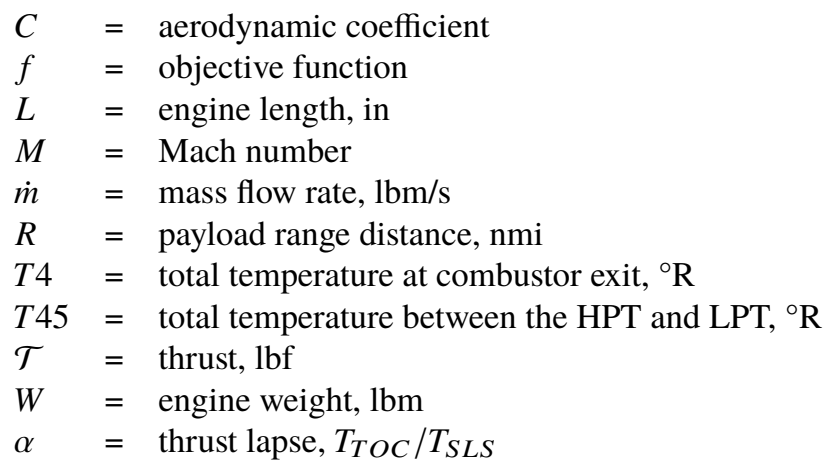

Subscripts

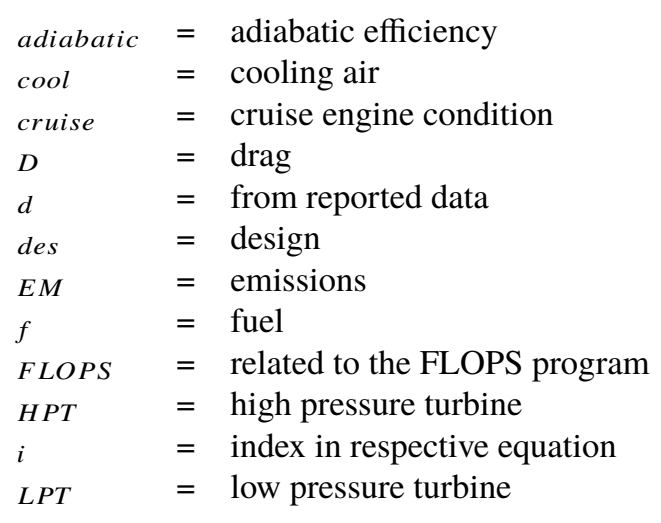

\footnotetext{
*Research Aerospace Engineer, Propulsion Systems Analysis Branch

${ }^{\dagger}$ Aerospace Engineer, Aeronautics Systems Analysis Branch
} 


$\begin{array}{ll}m & =\text { model output } \\ N P S S & =\text { related to the NPSS program } \\ n e t & =\text { net of gross and drag forces } \\ R T O & =\text { rolling takeoff operating condition } \\ \text { TOC } & =\text { top of climb operating condition } \\ S L S & =\text { sea level static operating condition } \\ W A T E & =\text { related to the WATE program }\end{array}$

\section{Introduction}

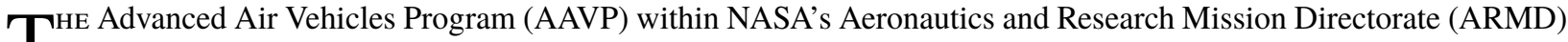
studies, evaluates, and explores future concepts that hold promise for revolutionary air-travel improvements. The Advanced Air Transport Technology (AATT) project under AAVP specifically focuses on advanced technologies and concepts for commercial subsonic aircraft. The technology goals of AATT are aimed at making improvements to aircraft noise, NOx emissions, and aircraft fuel/energy consumption [1]. While these goals have evolved over the years, the desired improvements are currently specified relative to a 2005 best in class baseline aircraft. Since this time, however, aviation technology has continued to advance and new commercial aircraft have been introduced. Therefore, a new baseline aircraft model is needed to better to establish the current technological capability of modern aircraft and guide the establishment of new research goals for future aircraft technology development.

The process for creating a new modern baseline model is a challenging task as it requires calibration to known data of an engine that represents today's industry. Historically, calibrating aircraft system performance at NASA has been done in a serial mode. The cycle decks, which capture engine performance throughout the flight envelope of an aircraft, were adjusted by changing the engine cycle model outside of the calibration of the airframe model. This was done repeatedly until an optimization of the aircraft system closely matched known data within some acceptable error. This process was time consuming and required a number of iterations. But by including the engine simulation in the calibration process of the airframe, closer matches to known data can be determined and a reduction in effort is possible. NASA is investigating the benefits of this simultaneous optimization process to generate a suitable engine/aircraft simulation model for the Boeing 737 (B737) MAX 8 aircraft using optimization methods.

The process of creating aircraft models has gone through recent improvements [2, 3] where the engine and aircraft performance simulations are simultaneously calibrated. Public data on engine and aircraft were calibrated simultaneously using random sampling of a range of calibration parameters. However, these efforts do not describe how the calibration procedure can be set up and conducted in an optimization problem. Presented in this paper is a Multi-Disciplinary Analysis and Optimization (MDAO) approach that automates the simultaneous calibration of airframe and engine models to publicly available information with a chosen optimizer. A model of the B737 MAX 8 and a LEAP-1B28 engine was generated using this process and the following sections outline the modeling approach. However, constructing an aircraft model is difficult for the researcher due to the proprietary nature of the engine and airframe designs. When generating aircraft simulations, proprietary data or information from any Original Equipment Manufacturer (OEM) limits the ability to generate models that reflect operational gas turbine engines and aircraft performance. The aircraft design details are proprietary but some guidance is given in this paper to predict OEM choices that impact performance. Characteristics such as engine air flow schemes, component efficiencies, pressure losses, operational temperatures, component pressure ratios, and the engine fan face Mach number are not made available to the general public. But by calibrating these parameters to match public data determines guesses of these unknown engine characteristics and provides appropriate combinations of a chosen set of parameters for aircraft performance for a given model [2]5]. The engine simulation and airframe models presented in the following sections were created using the Numerical Propulsion System Simulation (NPSS) [6] as the engine cycle program, the Weight Analysis of Turbine Engines (WATE) [7] as the engine weight program, and the Flight Optimization System (FLOPS) [8] as the airframe and aircraft performance program.

This paper describes the methodology to calibrate airframe and engine models to match an existing aircraft using limited public data. The procedure is first described as a general outline and then applied to the B737 MAX 8 aircraft as an example of a modern aircraft. Some guidance for accurate and reliable sources of data to create other aircraft models is included in the general outline section (Section III). All results presented in this paper for the B737 MAX 8 are intermediate optimization values and further work will be necessary to determine a final solution. 


\section{Calibration Procedure}

This section presents a framework for a new NASA process to simultaneously calibrate engine and airframe models for a complete aircraft system. The NPSS, FLOPS, and WATE programs are used in a MDAO tool for this study, as described in the following sections.

\section{A. Select Engine/Aircraft and Collect Available Public Data}

The first step in calibrating an aircraft model is to select an aircraft. This can be a time-consuming process; the researcher must chose among many particular aircraft design series and platforms. This includes both the airframe and engine combination which must match an actual operating aircraft. Then an extensive search of public data and information must be conducted to capture both engine and aircraft performance. The reader is directed to Ref. [9-12] for any aircraft design. The aircraft payload range diagram, an emissions data sheet, and a Type Certificate Data Sheet (TCDS) are the important components of information to obtain in order to follow the process laid out in this paper.

\section{B. Create Engine Cycle Model}

After the collection of public data, the engine cycle model architecture must be carefully created. There must be enough information accumulated to accurately represent an model the engine cycle. This would include bleed air locations in the compressor, the number of stages of the turbines and compressors, and any secondary airflow schemes, just to name a few. A typical turbofan cycle is shown in Figure 1 for a two-stream separate flow engine that can be adjusted for many engine cycles on commercial aircraft. The blue boxes represent components within an engine; the green boxes represent flows that enter or leave the engine; and the gold boxes represent the shafts of the engine.

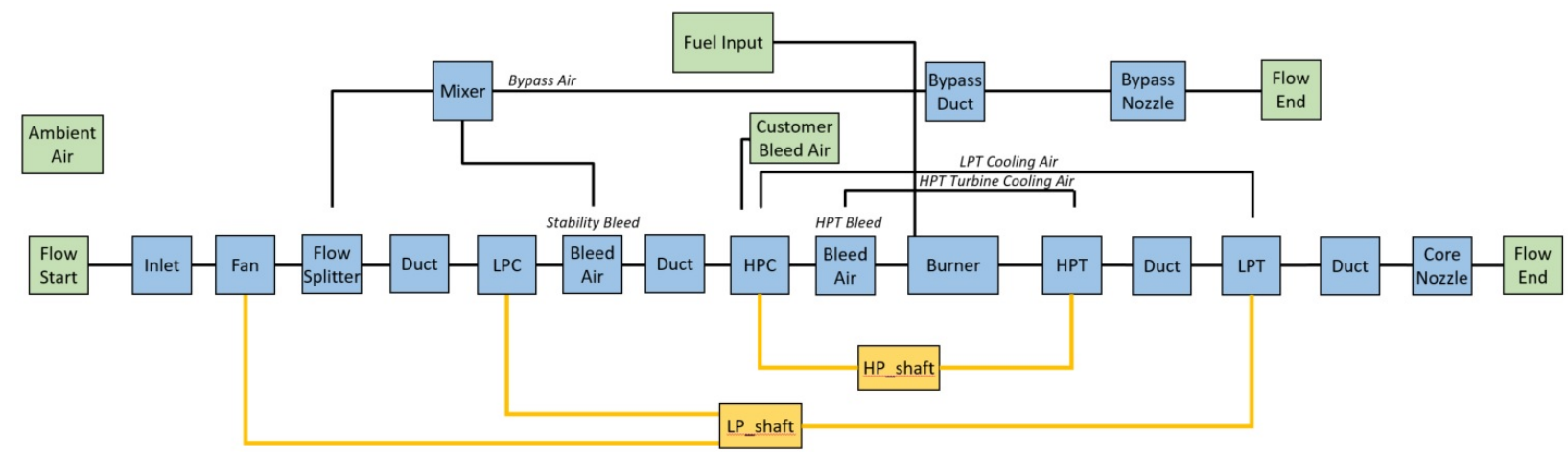

Fig. 1 Typical engine cycle model architecture

While not necessary, the process described in this paper uses a Multiple Design Point (MDP) approach [13] as displayed in Figure 2 to create the engine cycle. This approach allows a simultaneous solution between several operational points and ensures the cycle design satisfies performance requirements. These should be a combination of important aircraft operational points and engine static tests found from the data search in Section III.A. These are either common for engine design points or points that are readily found in the public domain. Other operation points can be included or excluded depending on available data found in a literature search. Figure 2 offers a suggested group of key operation points and static engine test data for the MDP. The array $\overline{\mathbf{X}}$ includes all the engine design scalars that are passed between all the operational points.

The suggested operation points for commercial aircraft are Top of Climb (TOC), Rolling Takeoff (RTO), Sea Level Static (SLS), and Cruise. The points that represent static engine test data that can be obtained in most literature searches are SLS and the engine emissions operation point from Ref. [10]. These are usually sufficient to capture the performance of many commercial transport aircraft.

\section{Create Airframe Model}

This step can simultaneously occur as the previous step in Section III.B. From the collection of all public data, the most important reference for the aircraft architecture and airframe model is the Airport Planning Document such as found in Ref. [12]. Commercial aircraft have an Airport Planning Document that typically includes maximum takeoff weight (MTOW), maximum landing weight (MLW), maximum zero fuel weight (MZFW), total fuel and a number of 


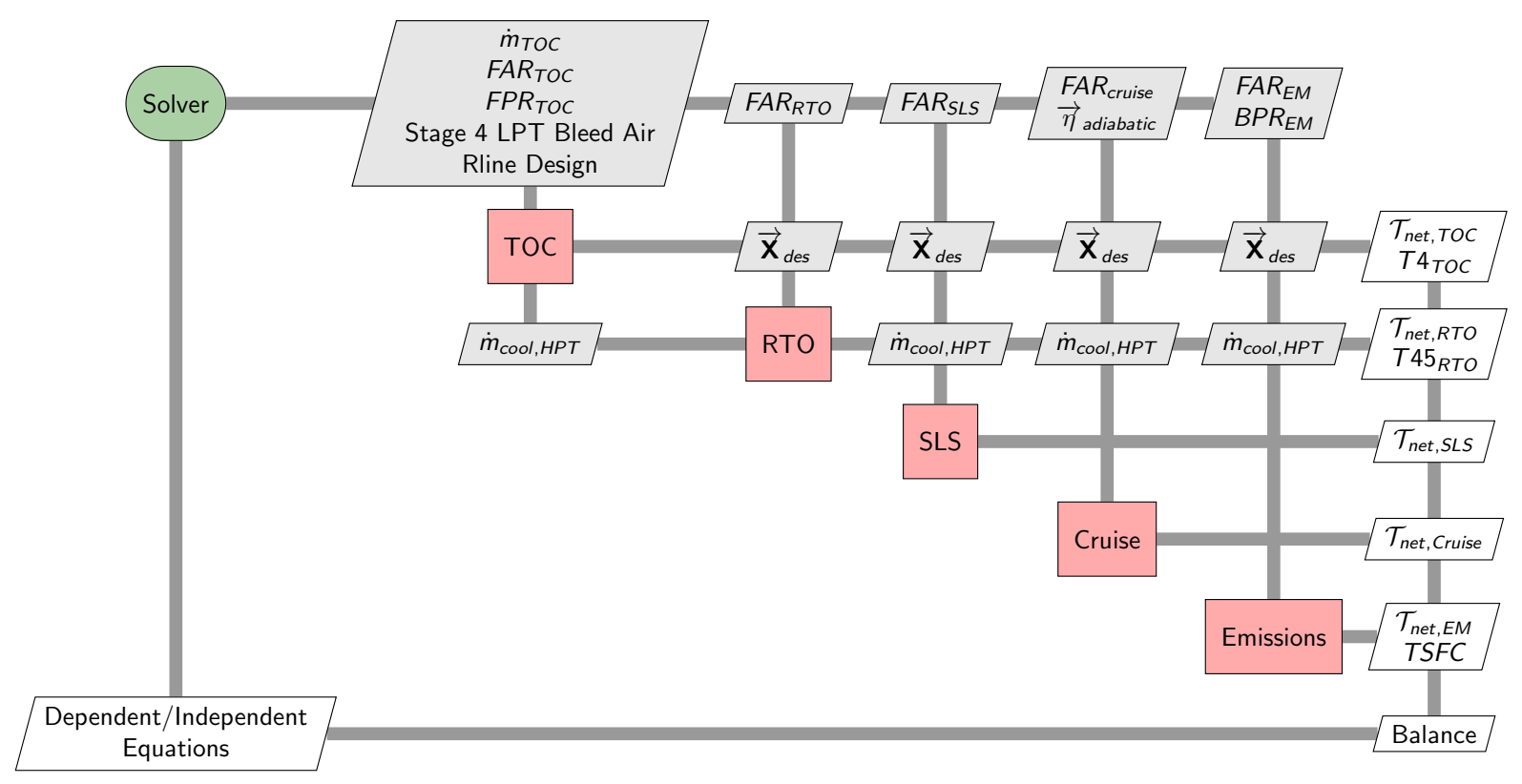

Fig. 2 Typical MDP architecture

three-view drawings. The aircraft model must include an estimation of the Operating Empty Weight (OEW). Though OEW may be reported in the Airport Planning Document, it varies with the configuration and is, at best, an estimation.

For example, the $\mathrm{OEW}$ is dependent on the number of seats on that particular aircraft, thus a B737 with a single-class configuration will have a different OEW than the same model with a two-class configuration. The benefit of using FLOPS as an airframe modeling software is that it has built-in routines to estimate weights, such as OEW, and performance metrics like payload and range. Part of this calibration effort is to control the aerodynamic parameters that enable researches to create a suitable aerodynamic model for the aircraft. The aerodynamic model is adjusted by controlling the aircraft drag until the aircraft is able to fly a prescribed range with a given payload, as taken from the payload-range diagram in the Airport Planning Document.

The aircraft weight and performance is modeled in FLOPS [8], a NASA-developed tool for estimating aircraft performance. This tool uses statistical models based on historical data for estimating component weights. The mission performance is calculated based on the engine performance and estimated aerodynamic properties. Input to FLOPS is through a text-based file in which users specify component weights and vehicle aerodynamics. The best approach to determine the dimensions of the aircraft is to create a three-dimensional computer model based on the three-view drawings of the Airport Planning Document [14]. This allows for specific dimensions to be measured in accordance with FLOPS definition of dimensions. For example, the wing area that FLOPS uses to calculate the aspect ratio and induced drag does not include the yehudi, but the yehudi may be included in a company's reported wing area. FLOPS requires numerous inputs to define the geometry, but a few major factors include the wing definition, empennage size, length of the passenger compartment and total length of the aircraft. The wing detail consists of listing the wing chord, thickness-to-chord and sweep across a series of spanwise sections. Obtaining these dimensions is easier with a user-developed computer model. Ref. [14] and [9] are commonly used sources to obtain approximate dimensions.

An estimation of the component weights from the defined geometry must be determined. Aircraft design books frequently include component weights for aircraft, which we can use as a guide. What is most important for FLOPS is that the overall weights, OEW and MTOW, match the modeled aircraft. When the mission analysis code is run, FLOPS assumes the aircraft is a point mass, thus the weight and balance is not critical. FLOPS has a series of "override" parameters to adjust component weights until the FLOPS predicted OEW matches the aircraft OEW.

FLOPS "flies" the mission profile using internally calculated aerodynamics based on the input geometry, with engine fuel flow data and thrust coming from the engine model. For a specific mission the takeoff gross weight (TOGW) is the sum of the OEW, payload and fuel. If the aircraft drag is too high or low, FLOPS under or over predicts the range for the mission. We can tune the aircraft model toward publicly available performance data by calibrating drag parameters 
to specific payload and range configurations. The default aerodynamic estimates within FLOPS estimate a subsonic transport's performance well, but FLOPS contains a number of calibration factors to fit the model to published data. For example, FLOPS maintains factors to control the amount of laminar flow on any surface, or control the "ellipticality" of the lift distribution along the wing. To reduce the design space, the model varied calibration factors scaling the viscous drag and induced drag using separate parameters, and an "airfoil technology parameter" that correlates with Mach drag divergence. This parameter has a lower limit of 1.0, which may correlate with a NACA four series airfoil and a Mach drag divergence near 0.6, whereas the upper limit of 2.0 may be a supercritical airfoil with a Mach drag divergence closer to 1 . Thus, in general, the higher the airfoil technology, the higher the Mach drag divergence.

\section{Define Calibration Parameters for Optimization}

Once the airframe and engine models are created, calibration parameters must be chosen. These are parameters that can impact the engine or aircraft performance such as, but not limited to, the engine Fan Pressure Ratio (FPR), TOC thrust, engine customer bleed air flow rates, induced drag on the aircraft, or engine component efficiencies. Selecting the right parameters that impact the engine cycle and aircraft performance is perhaps the most vital part of creating a realistic model. Any information important for engine and airframe modeling that isn't reported in the public domain is subject to calibration. Judgments must be made on the appropriate range for each calibration parameter. This is a process that may require several model iterations if the solution space does not contain a realistic engine or airframe design. When a parameter range cannot be determined then a random sampling of design parameters across a wide range is recommended before execution of an optimization to determine the limits on feasible solutions. The researcher is directed to Ref. [15, 16] for engine and airframe design to aid in determining calibration ranges that are appropriate for modern aircraft. However, investigating other references not included in this paper may be necessary.

\section{E. Define Objective Function for Optimization}

The objective function specifies how well the engine and aircraft model match public data, obtained in Section A. The model will closest match this data by varying the parameters selected in Section III.D to minimize the objective function. An aggregate of individual objective functions can be implemented to calibrate the model. These individual terms are known facts obtained in the literature search of Section III.A that represent system performance or characteristics. As an example, they can represent chosen payload range points, fuel flow values, engine weight, and engine length. Weighted coefficients in the aggregated objective function can be implemented depending upon the desire to match certain objectives over others. This would be important if information such as the payload range values were more important to match to data compared to other information such as engine fuel flow rates.

\section{F. Implement Models into a MDAO Problem}

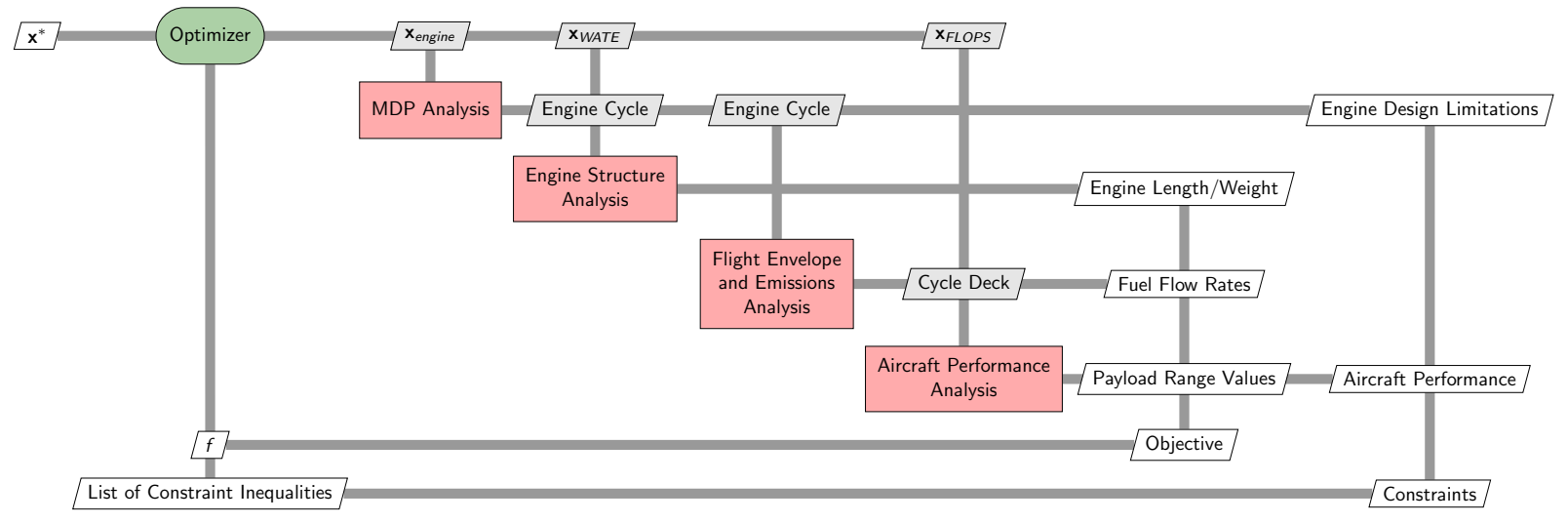

Fig. 3 MDAO architecture

After determining the objective function, a MDAO problem can be setup. This should be tailored so information necessary for the objective function calculation is extracted at appropriate points of an iteration. An essential part of a MDAO problem is communication between computer programs. Different computer programs can be combined 
to model the aircraft in this setup. The MDAO tool used in this paper is called OpenMDAO. OpenMDAO is the open-source high-performance computing tool developed at the NASA Glenn Research Center for systems analysis and multidisciplinary optimization [17]. It has a multitude of optimization schemes that can be applied to the problem set up by the user. Figure 3 illustrates the optimization process as applied to an aircraft, where evaluation of the objective function occurs at the end of each iteration. Constraints for the optimization can also be applied to limit the feasible solution space. These are constraints that limit the design of the engine or specify minimal aircraft performance during flight that are important for the overall design of the aircraft. In addition, random sampling of the design space is also possible via OpenMDAO if there is a need for an exploration of the design space to determine a range of a calibration parameter from Section III.D.

\section{G. Select Optimization Method}

Careful consideration must be given in the selection of an optimization scheme as well as its required settings for convergence. This may require tailoring the default settings in an MDAO platform such as OpenMDAO. This solution space for engine and airframe performance could be small with many local infeasible solution spaces with the applied constraints determined in Section F. The selected optimizer should be able to find the global optimum, with all applied constraints in the problem setup, in the presence of local optimums. For this reason, the selection of a non-gradient based optimizer is recommended. Many gradient-based optimization schemes may be subject to only finding a local optimization solution. However, missing the global best solution could also be an issue for non-gradient based schemes if the search in the solution space is too small. Once an optimizer is selected as appropriate to calibrating the aircraft model and all appropriate settings are applied, the optimization is ready execute to locate the global best solution.

\section{H. Execute Optimization and Evaluate Results}

The last step of the calibration procedure is to execute the optimization and evaluate the results. However, this may require the user to conduct model or MDAO setup corrections. Previous steps may also need to be repeated if the optimizer does not find a reasonable solution. Some optimization schemes are not guaranteed to locate a global optimum as iteration occurs. As setup in this MDAO problem, the objective function value should reduce in this problem setup to locate a global optimum.

Even if interrupted or prematurely stopped, important insights can still be obtained as initial results. These can include insights such as if calibration parameters are settling on specified values or wondering only to upper or lower limits. Ideally, the final values of the calibration should settle somewhere between the specified ranges without hitting an upper or lower calibration limit. If a parameter does hit an upper or lower limit then the engine cycle and airframe models should be carefully analyzed. It's possible the calibration ranges should be readjusted to a narrower/wider range or the parameter is not getting properly passed into the aircraft model. Ultimately, the researcher must decide if hitting a limit is acceptable in the final solution or restart the optimization with a wider range.

Evaluation of the error between the model and public data can determine if the model is adequately matching the data obtained in Section III.A. Once a final solution is acceptable, calibration of the aircaft system model is complete.

\section{Calibration Procedure of the B737 MAX 8 Aircraft}

As an initial demonstration of this process, the B737 MAX 8 was selected as a calibration aircraft for study to represent a modern design. This is anticipated to be an improved process at NASA to model a baseline aircraft system to study and assess new engine and airframe technology. This is a preliminary analysis that will be refined further in future work. Therefore, final calibration results will not be reported in this paper.

\section{A. Select Engine/Aircraft and Collect Available Public Data}

The International Civil Aviation Organization (ICAO), the Federal Aviation Administration (FAA), and other regulating authorities release regulatory data on commercial aircraft. This study selected data from an ICAO emission databank sheet [18] and a FAA TCDS [19] for the chosen engine of the B737 MAX 8, the LEAP-1B28. Other international regulating authorities release the same information in different formats as the TCDS [20, 21], but the information presented by the FAA is sufficient for this study for an engine cycle model. Table 1 lists the information extracted from all the collected sources from the literature search to model this aircraft.

Due to low speed limitations in the component performance maps being used, the NPSS cycle simulation had difficulty converging at the idle power condition from Ref. [18]. Since there were a significant number of other flight 
Table 1 Relevant Data for the LEAP-1B28 Engine

\begin{tabular}{|c|c|c|}
\hline Parameter & Value & Description/Notes \\
\hline Takeoff rating/rated output & $29,317 \mathrm{lbf}$ & Uninstalled thrust [Ref.[18, 19]] \\
\hline Takeoff flat rating ambient temperature & $545.67^{\circ} \mathrm{R}$ & At sea Level [Ref. [19]] \\
\hline Max N1 (Low Pressure Spool) speed & 4,596 RPM (104.3\% ) & $\begin{array}{l}\text { Used to acquire } 100 \% \text { shaft speed } \\
\text { [Ref.[19]] }\end{array}$ \\
\hline Max N2 (High Pressure Spool) speed & 21,171 RPM (117.5\% ) & $\begin{array}{l}\text { Used to acquire } 100 \% \text { shaft speed } \\
\text { [Ref.[19]] }\end{array}$ \\
\hline Engine weight & $6,128 \mathrm{lbm}$ & Weight of the basic engine [Ref.[19]] \\
\hline Engine length & 123.9 in & $\begin{array}{l}\text { Fwd. fan case flange to turbine } \\
\text { rear vane aft flange [Ref.[19]] }\end{array}$ \\
\hline Bypass ratio & 8.6 & At sea level for emissions [Ref. [18]] \\
\hline Overall pressure ratio & 41.5 & At sea Level for emissions [Ref. [18]] \\
\hline Takeoff fuel flow, $\dot{m}_{f, r, 1}$ & $2.34 \mathrm{lbm} / \mathrm{s}$ & $\begin{array}{l}100 \% \text { of max thrust [Ref. [18]] } \\
\text { at sea level for emissions }\end{array}$ \\
\hline Climb out fuel flow, $\dot{m}_{f, r, 2}$ & $1.91 \mathrm{lbm} / \mathrm{s}$ & $\begin{array}{l}85 \% \text { of max thrust [Ref. [18]] } \\
\text { at sea level for emissions }\end{array}$ \\
\hline Approach fuel flow, $\dot{m}_{f, r, 3}$ & $0.61 \mathrm{lbm} / \mathrm{s}$ & $\begin{array}{l}30 \% \text { of max thrust [Ref. [18]] } \\
\text { at sea level for emissions }\end{array}$ \\
\hline Fan diameter & 69.29 in & Ref.[22] \\
\hline Compressor stages (fan, LPC, HPC) & $1+3+10$ & $\begin{array}{l}1 \text { stage fan, } 3 \text { stage LPC (booster), } \\
10 \text { stage HPC [Ref. [23]] }\end{array}$ \\
\hline Turbine stages (HPT, LPT) & $2+5$ & 2 stage HPT, 5 stage LPT [Ref. [23]] \\
\hline
\end{tabular}

conditions already identified, it was excluded from the calibration procedure. This made for a closer match of aircraft performance and engine fuel flow during calibration.

As an important note, the thrust ratings in the TCDS are reported values for an engine uninstalled from the aircraft, fitted with a production nozzle and a bellmouth inlet on an appropriate test stand for certification, corrected to Standard Day conditions. This means the performance of the engine installed on an aircraft will be different. However, for this initial study installation effects are assumed to be negligible and the reported TCDS thrust data was used as installed aircraft data. Another important note is the emissions data sheet reports data with zero customer horsepower extraction off of the engine accessory gearbox, corrected to Standard Day conditions. These notes are accurately implemented in the respective MDP operation points.

Table 2 lists the set of missions used for the calibration effort of the aircraft model. The calibration points are digitized from the payload-range diagram published in the Airport Planning Document [14]. The values in Table 2 are listed down to the pound, but this is far more accurate than the actual data. Digitizing the payload-range diagram introduces error, and the payload-range diagram is only representative of a single configuration. Despite these limitations, the FLOPS wrapper uses these values within its objective function, as described in Section IV.E. The OEW is reported as $99,360 \mathrm{lbm}$ in Ref [14]. This will greatly reduce the modeling efforts for the airframe. See Section IV.C for OEW important information regarding the implementation into the airframe model.

\section{B. Create Engine Cycle Model}

The selected engine is modeled in NPSS using the architecture shown in Figure 1 This model captures the level of fidelity needed to assess a system level model of engine performance. The customer bleed air depicted in Figure 1 will come from stage 4 in the High Pressure Compressor (HPC), assumed to be the only source of customer bleed air for normal operation. Many detailed features were found in the literature search for bleed air and secondary air flow. For simplicity, the features were left out of the model are active clearance control around the turbines, modulated cooling air 
Table 2 Selected Payload Range Data

\begin{tabular}{cccc}
\hline Payload Range Description & $\begin{array}{c}\text { Range (nmi) } \\
\boldsymbol{R}_{\boldsymbol{d}, \mathbf{1}-\mathbf{1 0}}\end{array}$ & $\begin{array}{c}\text { OEW Plus } \\
\text { Payload (lbm) }\end{array}$ & Fuel (lbm) \\
\hline Design Mission & 3379 & 136,752 & 44,448 \\
Max TOGW/payload, partial fuel & 2528 & 145,447 & 35,753 \\
Max TOGW, partial payload, max fuel & 3465 & 135,938 & 45,262 \\
Zero Payload and max fuel & 4385 & 99,360 & 45,694 \\
170k TOGW, partial payload, partial fuel & 2017 & 141,038 & 28,962 \\
170k TOGW, partial payload, partial fuel & 3019 & 131,174 & 38,826 \\
155k TOGW, partial payload, partial fuel & 1516 & 132,877 & 22,123 \\
155k TOGW, partial payload, partial fuel & 3019 & 118,924 & 36,076 \\
140k TOGW, partial payload, partial fuel & 1516 & 119,633 & 20,367 \\
140k TOGW, partial payload, partial fuel & 3019 & 106,694 & 33,306 \\
\hline
\end{tabular}

for the HPT blades and vanes, control logic for switching between HPC Stage 4 and 10 customer bleed air locations, and HPC Stage 7 engine anti-icing air.

A Multiple Design Point (MDP) approach [13] was used in NPSS to match all reported public data to create the thermodynamic cycle. The key points of operation for this design are TOC, RTO, SLS, Cruise, and the emissions certification point. These are outlined in detail in Table 3 along with the atmospheric conditions for each operational point that are typical for engine design [15]. The emissions operation point captures the conditions reported in the ICAO Emissions Databank Sheet [18]. Figure 2 displays the MDP approach with inputs and outputs of each operational point. The primary thermodynamic cycle design point was assumed to be at TOC but cooling flow requirements for the HPT blades and vanes were determined by RTO conditions. RTO is typically the point of operation where metal temperatures are the hottest in this flight envelope and cooling requirements were determined here.

Table 3 MDP Operational Points

\begin{tabular}{ccccc}
\hline Description & Altitude [ft] & Atmosphere & $\boldsymbol{M}$ & $\mathcal{T}[\mathrm{lbf}]$ \\
\hline Top of Climb (TOC) & 37,000 & Standard Day & 0.79 & $\alpha \mathcal{T}_{S L S}$ \\
Rolling Takeoff (RTO) & 0 & Hot Day & 0.25 & $\mathcal{T}_{S L S} / 1.2553$ \\
Sea Level Static (SLS) & 0 & Hot Day & 0.0 & 29,317 \\
Cruise & 37,000 & Standard Day & 0.79 & $0.9 \mathcal{T}_{T O C}$ \\
ICAO Emissions Test & 0 & Standard Day & 0.0 & 29,317 \\
\hline
\end{tabular}

Thrust targets for RTO, Cruise, and TOC are not found in reported data. The thrust for RTO is predicted using the Boeing Equivalent Thrust at Mach 0.25 in Equation 1 from the published SLS thrust of the engine displayed in Table 3 The thrust target for TOC is determined by a thrust lapse factor $\alpha$, the ratio of TOC thrust to SLS thrust. The cruise throttle setting is assumed to be $90 \%$ of the TOC thrust.

$$
\mathcal{T}_{S L S}=1.2553 \mathcal{T}_{\text {RTO }}
$$

\section{Create Airframe Model}

Figure 4] shows the model created from Ref. [14] of the B737 MAX 8. The aircraft's mission profile is specified in a FLOPS input file to match all the physical characteristics given in Ref. [14]. The B737 MAX 8 flies a typical aircraft mission profile shown in Fig. 5. consisting of a primary mission and a reserve mission in the event the aircraft is diverted or required to hold before landing. The analysis investigates various points on a payload-range diagram from Table 2, thus the cruise distance varies depending on the specific mission, but the other aspects of the flight remain constant. Figure 5 shows a step-cruise profile to match current operation procedures, with FLOPS determining when the aircraft should move up in altitude. 

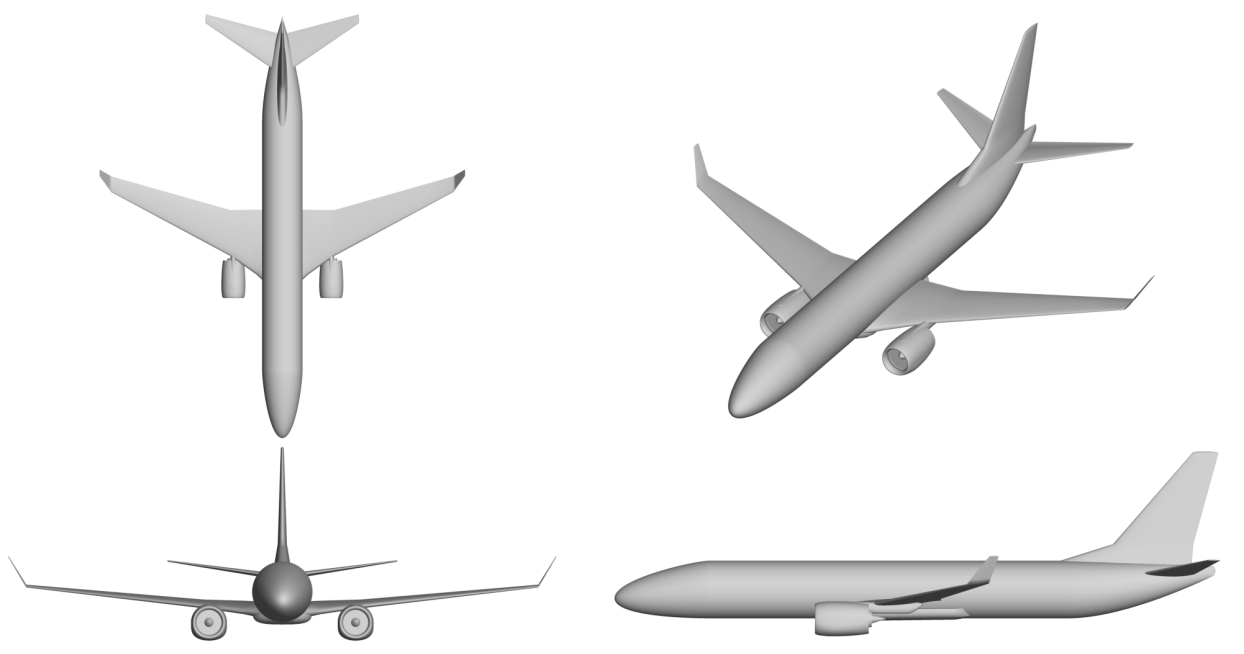

Fig. 4 OpenVSP [24] render of the selected aircraft used to define the geometric inputs to FLOPS.

The model of the airframe was simplified because the OEW was given in Ref. [14] of 99,360 lbm. Therefore, each payload range point in the analysis does not require an OEW model prediction. This greatly reduced modeling efforts by directly inputting the gross weight of the aircraft at the start of each payload range mission as an aggregate of payload, OEW, and fuel.

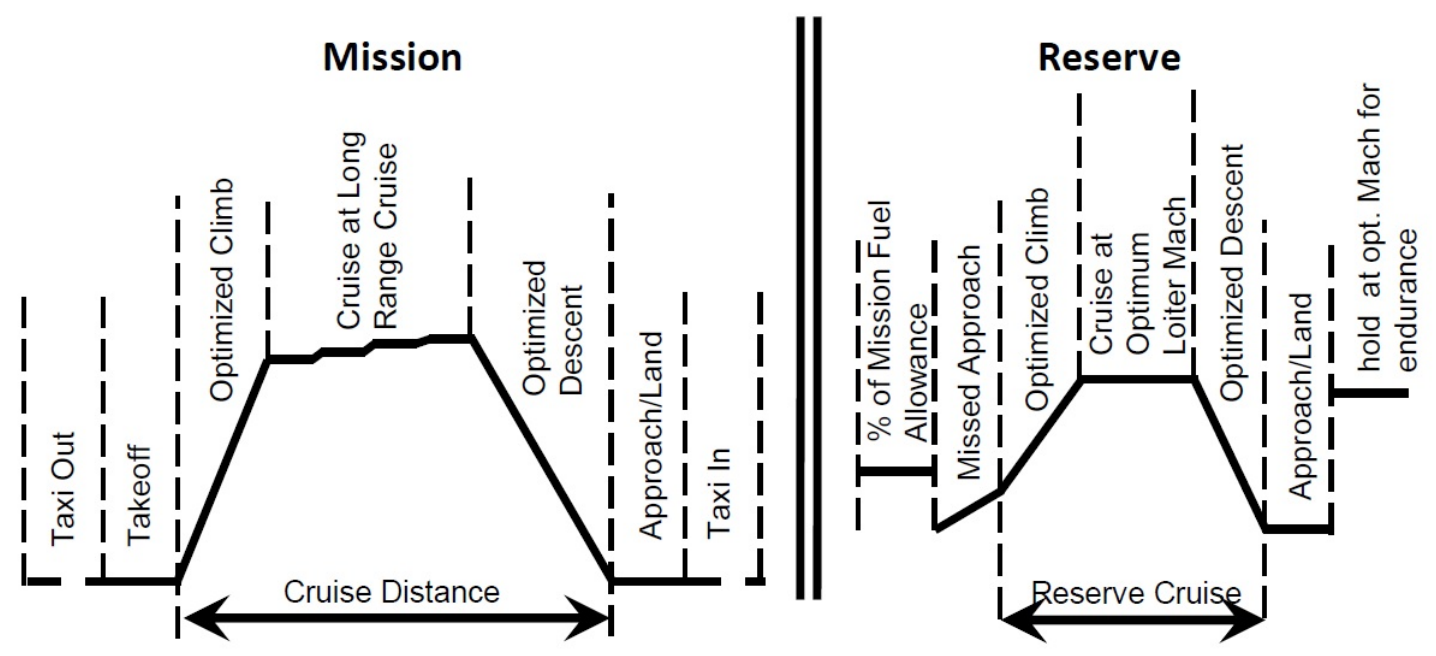

Fig. 5 Mission profile for the cruise mission. The cruise range varies with mission.

\section{Define Calibration Parameters for Optimization}

The selected design calibration variables are listed in Table 4 These parameters and their allowable ranges were chosen as initial estimates based upon experience and available information. The literature search presented some insights in the limits for HPC stage 4 customer bleed air, T45 RTO temperature limits, and HPC pressure ratio. HPC stage 4 HPC customer bleed air is mentioned to have a maximum of 10\% in Ref. [19]. T45 RTO Temperature is mentioned to have a maximum limit of $2360{ }^{\circ} \mathrm{R}$ in Ref. [19]. The HPC Pressure Ratio reported in Ref. [23] is 22 but is allowed to vary as a calibration parameter over a small range because of the large impact this parameter has on the cycle. 
Table 4 Design Calibration Variables

\begin{tabular}{lc}
\hline Variable & Calibration Range \\
\hline Stage 4 HPC customer bleed air & $0.0-10.0 \%$ \\
Stage 4 bleed air for LPT cooling & $0.02-0.12$ \\
LPT air split factor between chargeable & $0.5-1.65$ \\
and nonchargeable air & \\
Fan polytropic efficiency & $0.87-0.96$ \\
LPC polytropic efficiency & $0.90-0.95$ \\
HPC polytropic efficiency & $0.90-0.95$ \\
HPT polytropic efficiency & $0.89-0.94$ \\
LPT polytropic efficiency & $0.90-0.95$ \\
TOC FPR & $1.5-1.6$ \\
Combustor pressure drop & $4.0-6.5 \%$ \\
Bypass duct pressure drop & $1.0-2.5 \%$ \\
HPT metal temperature of blade and vanes & $2260-2660{ }^{\circ} \mathrm{R}$ \\
T45 RTO temperature & $2260-2360{ }^{\circ} \mathrm{R}$ \\
HPC pressure ratio & $21.5-22.4$ \\
HPC stage length per stage & $3.00-5.00$ inches \\
LPT stage length per stage & $3.75-5.50$ inches \\
Thrust lapse factor $\alpha$ & $0.16-0.20$ \\
Wing technology factor & $1.7-1.9$ \\
Aircraft induced drag & $0.9-1.2$ \\
Aircraft viscous drag & $0.9-1.2$ \\
\hline
\end{tabular}

\section{E. Define Objective Function for Optimization}

The objective function created for the B737 MAX 8 aircraft matches fuel flow, payload range, engine weight, and engine length data. Equation 2 shows the overall objective function $f$ as an aggregate of equally weighted components from Equations 3, 4 and 5. An assessment of the objective function revealed these components were of similar orders of magnitude, thus equal weighting was acceptable for the initial optimization study presented here. These objective functions utilize fuel flow rates $\dot{m}_{f}$, payload range points $R$, engine weight $W$ (reported as $6128 \mathrm{lbm}$ in Ref. [19]), and engine length $L$ (reported as 123.9 in in Ref. [19]). The subscripts $m$ and $d$ represent model output or reported data values, respectively. It should be noted that since the OEW of the aircraft is reported, the engine pod weight predicted in the WATE tool has no impact on the aircraft optimization.

$$
\begin{gathered}
f=\frac{1}{3} f_{N P S S}+\frac{1}{3} f_{F L O P S}+\frac{1}{3} f_{W A T E} \\
f_{N P S S}=\frac{1}{3} \sum_{i=1}^{3} \frac{\left(\dot{m}_{f, d, i}-\dot{m}_{f, m, i}\right)^{2}}{\dot{m}_{f, d, i}^{2}} \\
f_{F L O P S}=\frac{1}{10} \sum_{i=1}^{10} \frac{\left(R_{d, i}-R_{m, i}\right)^{2}}{R_{d, i}^{2}} \\
f_{W A T E}=\frac{1}{2} \frac{\left(6128-W_{m}\right)^{2}}{6128^{2}}+\frac{1}{2} \frac{\left(123.9-L_{m}\right)^{2}}{123.9^{2}}
\end{gathered}
$$




\section{F. Implement Models into a MDAO Problem}

Figure 3 shows the OpenMDAO optimization environment. Each iteration executes the cycle design in NPSS, the engine weight and length in WATE, the flight envelope and emissions of the engine in NPSS, and finally the aircraft missions performance in FLOPS. The output of each cycle design is used to determine fuel flow data points and engine performance for the entire flight envelope. After the engine cycle is created, the WATE program determines the engine length and weight. Finally, the FLOPS program runs and a set of payload range values are generated. The results of the analysis are compared to the reported values listed in Tables 1 and 2 and constrained to the TOC fan face Mach number and aircraft climb rate. The constraints applied to the aircraft model are listed in Table 5

Table 5 Constraints for the B737 MAX 8

\begin{tabular}{lc}
\hline Variable & Calibration Range \\
\hline Fan Face Mach Number at TOC & $0.60<$ Mach Number $<0.685$ \\
Potential Climb Rate at 35k ft for the Design Mission & $\geq 300 \mathrm{ft} / \mathrm{min}$ \\
\hline
\end{tabular}

\section{G. Select Optimization Method}

The chosen optimizer for an initial study is the Augmented Lagrangian Particle Swarm Optimizer (ALPSO). Prior research has shown that an ALPSO optimization scheme can produce equivalent or better objective values compared to other optimization schemes [25]. ALPSO is a non-gradient based scheme originally inspired by the coordinated movement of flocks of birds and schools of fish [26]. Non-gradient optimizers are less influenced by local maximums in a solution space or by local infeasible solution spaces. What makes ALPSO unique compared to a regular Particle Swarm Optimization (PSO) is the objective function and inequality constraints become an unconstrained optimization problem via a Lagrangian transformation, allowing the implementation of the inlet Mach number and aircraft performance constraints listed in Table 5 that would otherwise not be allowed in a regular PSO. A set of calibration variables represents an engine design as a particle that iterates according to cognitive and social behavior. The swarm is a predefined set of particles (the swarm size) that will iterate throughout the optimization according to the swarm's best global position and the particle's best local position, as evaluated by the objective function. It was important to select a swarm size large enough for an exhaustive search for the global best position. This study used a recommended practice of a swarm size ten times greater than the number of calibration variables. Table 6 displays key parameters for the ALPSO driver that were changed from the default settings of the optimization driver in OpenMDAO.

Table 6 ALPSO Parameter Adjustments in OpenMDAO

\begin{tabular}{ccl}
\hline Parameters & Chosen Value & Comments \\
\hline SwarmSize & 200 & $10 \times$ Number of dimensions \\
itol & $1 \mathrm{e}-8$ & Inequality convergence tolerance \\
rtol & $1 \mathrm{e}-7$ & Relative convergence tolerance \\
atol & $1 \mathrm{e}-7$ & Absolute convergence tolerance \\
dtol & $1 \mathrm{e}-5$ & Relative tolerance in particles distance to terminate \\
\hline
\end{tabular}

\section{H. Execute Optimization and Evaluate Results}

As an initial demonstration, the ALPSO optimizer ran for over three outer iterations, consisting of 3,800 particle runs. As observed from Table 7, a reduction in the global best objective function was discovered at the end of each iteration. The global distance reduction of each outer iteration also suggests the ALPSO swarm is narrowing down to a best global solution. The selected set of calibration variables after more than three outer iterations produce aircraft model results that closely match publicly available data, as shown in Tables 8.9 , and 10 The payload range values compared to the data from Ref. [14] is displayed in Figure 6 The smallest error observed is reported in Table 9, suggesting the NPSS thermodynamic model is closely predicting engine operation at the emissions certification operation point. Other values show an error below $2 \%$ for all cases in Tables 8,9 , and 10 . These initial results show good agreement with public data. 
Table 7 ALPSO Driver Iteration Outputs

\begin{tabular}{ccc}
\hline Major Iteration & Best Objective Value Found & Global Distance \\
\hline 1 & 0.000246 & 2.0014 \\
2 & 0.000212 & 0.9452 \\
3 & 0.000139 & 0.9220 \\
\hline
\end{tabular}

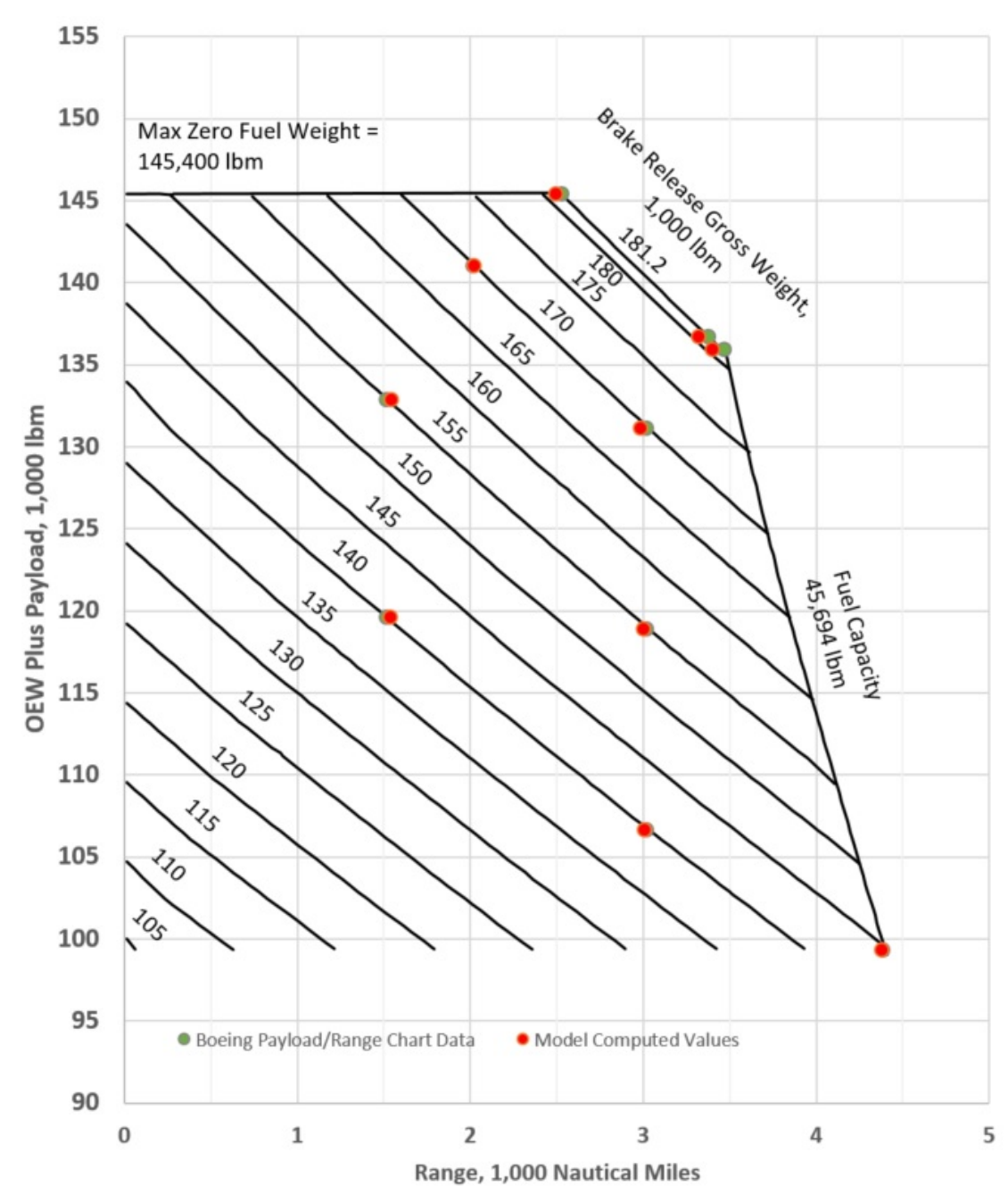

Fig. 6 Payload range diagram [reproduced from Ref. [14]] with initial results

\section{Concluding Remarks and Future Work}

The AATT project is seeking new engine and airfarme baseline models representative of current state-of-the-art technology. An improved process was desirable to an create aircraft model for an evaluation of best-in-class technology intended as an update to the previous 2005 baseline. This improved process uses a set of simulation tools coupled with an optimizer to drive the simulation inputs towards a desired solution based on publicly available data. The B737 MAX 8 aircraft was selected as the initial study case subjected to optimization. During the progress of the optimization, the ALPSO driver output showed a global best solution exists due to a reduction of the global best objective function value of the swarm and the reduced global distance of particles, as reported at each outer iteration. This justifies the application of an optimizer to find a global best position in a solution space is a valid method in creating an aircraft model. This is potentially an improvement over a process that required information to be manually passed between codes and engineers. 
Table 8 Payload Range Initial Results in Nautical Miles for Lowest Objective Value

\begin{tabular}{cccc}
\hline Payload Range Description & Reported Value & Computed Value & Error \\
\hline Design Mission & 3379 & 3319.5 & $-1.76 \%$ \\
Max TOGW/payload, partial fuel & 2528 & 2495 & $-1.31 \%$ \\
Max TOGW, partial payload, max fuel & 3465 & 3398.9 & $-1.91 \%$ \\
Zero payload and max fuel & 4385 & 4375.8 & $-0.21 \%$ \\
170k TOGW, partial payload, partial fuel & 2017 & 2022.1 & $0.25 \%$ \\
170k TOGW, partial payload, partial fuel & 3019 & 2985.7 & $-1.10 \%$ \\
155k TOGW, partial payload, partial fuel & 1516 & 1545.5 & $1.95 \%$ \\
155k TOGW, partial payload, partial fuel & 3019 & 3000.7 & $-0.61 \%$ \\
140k TOGW, partial payload, partial fuel & 1516 & 1535.4 & $1.28 \%$ \\
140k TOGW, partial payload, partial fuel & 3019 & 3007.4 & $-0.38 \%$ \\
\hline
\end{tabular}

Table 9 Fuel Flow Initial Results to Match ICAO Emissions for Lowest Objective Value

\begin{tabular}{ccccc}
\hline Mode & Power Setting & Fuel Flow Data $[\mathbf{l b m} / \mathbf{s}]$ & Fuel Flow Model $[\mathbf{l b m} / \mathbf{s}]$ & Error \\
\hline Take-Off & 100 & 2.339 & 2.345 & $0.25 \%$ \\
Climbout & 85 & 1.905 & 1.917 & $0.66 \%$ \\
Approach & 30 & 0.611 & 0.615 & $0.72 \%$ \\
\hline
\end{tabular}

Table 10 Engine Weight and Length Initial Results for Lowest Objective Value

\begin{tabular}{cccc}
\hline & TCDS Data & Model Results & Error \\
\hline Engine weight $(\mathrm{lbm})$ & 6128 & 6024.6 & $-1.69 \%$ \\
Engine length (inches) & 123.9 & 125.5 & $1.29 \%$ \\
\hline
\end{tabular}

The improved procedure outlined in this paper became the framework for the creation of a B737 MAX 8 aircraft model. Further study of the optimized solution is necessary to determine the model is representative of a real aircraft design. The reported OEW of the aircraft simplified the airframe portion of the model, requiring less effort to predict aircraft performance. This allows the removal of the WATE program from the analysis, potentially reducing optimization time for future efforts. For the engine model, careful attention was given in the research step of the process to locate bleed port locations, apply inlet geometry, and apply stability bleed for engine operation for an accurate cycle model but other future refinements may be necessary. Evaluation of the initial results required a number of refinements to the model and MDAO problem setup, making feedback an important part of the process for all steps of the procedure. This MDAO setup still needs some further refinement to reduce optimization time, such as taking advantage of excluding the WATE analysis from the optimizer. Other non-gradient based optimization schemes besides ALPSO can also be investigated in future work to determine if better or equivalent objective values can be obtained.

When using the process outlined in this paper for any aircraft application, caution must be used in accepting the final outcome without a close analysis of the calibration results. Involvement of an experienced engineering team is still vital to this modeling process and shouldn't be substituted by an optimizer. A calibrated solution found by any optimization scheme is not guaranteed to reflect a realistic aircraft design and further refinement may be necessary. If a user desires to implement other aircraft modeling tools, such as other cycle/airframe modeling programs, then these can be included or substituted with adjustments. These other tools must have the capability to function within an MDAO problem setup to allow calibration to known data. Refinements to this process may also be necessary.

\section{Acknowledgments}

The authors thank AATT for funding and supporting this research. The authors appreciate the mentoring and direction from Jeff Berton, Justin Gray, Scott Jones, Eric Hendricks, and Mike Tong from NASA Glenn Research Center. 


\section{References}

[1] “Advanced Air Transport Technology (AATT) Project,” NASA, Aug 2017. URL https://www.nas.nasa.gov/assets/ pdf/ams/2017/AMS_20170808_Heidmann.pdf

[2] Barros, P., Kirby, M. R., and Mavris, D., "An Approach for Verification and Validation of the Environmental Design Space," 26th International Congress of the Aeronautical Sciences, AIAA, Anchorage, Alaska, 2008. doi:10.2514/6.2008-8875.

[3] Kirby, M. R., and Mavris, D. N., "The Environmental Design Space," 26th International Congress of the Aeronautical Sciences, AIAA, Anchorage, Alaska, 2008. ICAS2008-4.7.3 .

[4] Hearn, T., Hendricks, E., Chin, J., Gray, J., and Moore, K. T., "Optimization of Turbine Engine Cycle Analysis with Analytic Derivatives," AIAA Aviation 2016 Conference, GRC-E-DAA-TN32054, AIAA, Washington, DC, 2016.

[5] Espana, M. D., and Gilyard, G. B., "On the estimation algorithm used in adaptive performance optimization of turbofan engines," AIAA Joint Propulsion Conference, AIAA, Monterey, CA, 1993.

[6] Claus, R. W., Evans, A. L., Lytle, J. K., and Nichols, L. D., "Numerical Propulsion System Simulation," Computing Systems in Engineering, 1991, pp. 357-364. doi:10.1016/0956-0521(91)90003-N.

[7] Tong, M. T., and Naylor, B. A., “An Object-Oriented Computer Code for Aircraft Engine Weight Estimation,” American Society of Mechanical Engineers, Berlin, Germany, 2008. doi:10.1115/GT2008-50062.

[8] McCullers, L. A., “Aircraft Configuration Optimization Including Optimized Flight Profiles,” NASA CP-2327, April 1984, pp. $396-412$.

[9] Jane's Information Group, Jane's All the World's Aircraft, Information Handling Services, Coulson, Surrey, UK; Alexandria, Virginia, 2018-2019. ISSN 0075-3017.

[10] “ICAO Aircraft Engine Emissions Databank,” EASA, May 2018. URL https://www.easa.europa.eu/node/15672

[11] “Type Certificate Data Sheets,” FAA, Dec 2018. URL http://rgl.faa.gov/Regulatory_and_Guidance_Library/ rgMakeModel.nsf/MainFrame?OpenFrameset.

[12] “Airplane Characteristics for Airport Planning," Boeing, Mar 2019. URL https://www.boeing.com/commercial/ airports/plan_manuals.page.

[13] Schutte, J. S., "Simultaneous Multi-Design Point Approach To Gas Turbine On-Design Cycle Analysis For Aircraft Engines," Ph.D. thesis, The Georgia Institute of Technology, Altanta, GA, May 2009.

[14] 737 MAX Airplane Characteristics for Airport Planning, Boeing, Jun 2018. Revision A; Document Number: D6-38A004.

[15] Mattingly, J. D., Heiser, W. H., and Pratt, D. T., Aircraft Engine Design, $2^{\text {nd }}$ ed., AIAA Education Series, AIAA, Reston, VA, 2002.

[16] Roskam, J., Part I: Preliminary Sizing of Airplanes, Airplane Design, DARcorporation, Lawrence, Kansas, 2015.

[17] Gray, J. S., Hwang, J. T., Martins, J. R. R. A., Moore, K. T., and Naylor, B. A., "OpenMDAO: An Open-Source Framework for Multidisciplinary Design, Analysis, and Optimization," Structural and Multidisciplinary Optimization, Vol. 59, 2019, pp. 1075-1104. doi:10.1007/s00158-019-02211-z.

[18] Engine Exhuast Emissions Data Bank - Subsonic Engines, International Civil Aviation Organization, Sep 2013. Engine Identification: LEAP-1B28; Unique ID Number: 18CM084.

[19] Type Certification Data Sheet E00088EN, Federal Aviation Administration, Jun 2017. Revision 2.

[20] Type Certification Data Sheet No. E.115, European Aviation Safety Agency, Mar 2018.

[21] Type Certification Data Sheet No. EM-2018T04-00, Agência Nacional de Aviação Civil, Apr 2018.

[22] Teal, M., “New 737 MAX: Improved Fuel Efficiency and Performance,” Boeing AERO Magazine, Q1 2014.

[23] LEAP-1B, Boeing 737 MAX, CFM, Jun 2017. Digital Engine Brochure.

[24] Hahn, A., "Vehicle Sketch Pad: A Parametric Geometry Modeler for Conceptual Aircraft Design," 48th AIAA Aerospace Sciences Meeting Including the New Horizons Forum and Aerospace Exposition, AIAA, Orlando, Florida, 2010. doi:10.2514/6.2010-657, aIAA 2010-657. 
[25] Perez, R. E., and Jansen, P. W., "Constrained structural design optimization via a parallel augmented Lagrangian particle swarm optimization approach,” Computers and Structures, Vol. 89, 2011, pp. 1352-1366. doi:10.1016/j.compstruc.2011.03.011.

[26] Kennedy, J., and Eberhart, R., “Particle Swarm Optimization,” IEEE, Perth, Western Australia, Australia, 1995, pp. $1942-1948$. doi:10.1109/ICNN.1995.488968. 